\title{
Mortality among Cancer Patients within 90 Days of Therapy in a Tertiary Hospital, Tanzania: Is Our Pretherapy Screening Effective?
}

\author{
Marygoreth J. Changalucha, ${ }^{1}$ Martha F. Mushi $\mathbb{D}^{2},{ }^{2}$ Rodrick Kabangila, ${ }^{1,3}$ Vitus Silago, ${ }^{2}$ \\ Beda Likonda, ${ }^{4}$ and Stephen E. Mshana ${ }^{2}$ \\ ${ }^{1}$ Department of Internal Medicine, Bugando Medical Centre/Catholic University of Health and Allied Sciences, Mwanza, Tanzania \\ ${ }^{2}$ Department of Microbiology and Immunology, Catholic University of Health and Allied Sciences, Mwanza, Tanzania \\ ${ }^{3}$ Kamanga Medics Hospital, Mwanza, Tanzania \\ ${ }^{4}$ Oncology Department, Bugando Medical Centre/Catholic University of Health and Allied Sciences, Mwanza, Tanzania
}

Correspondence should be addressed to Martha F. Mushi; mushimartha@gmail.com

Received 22 April 2020; Revised 22 July 2020; Accepted 31 July 2020; Published 12 August 2020

Academic Editor: Eleanor Kane

Copyright (c) 2020 Marygoreth J. Changalucha et al. This is an open access article distributed under the Creative Commons Attribution License, which permits unrestricted use, distribution, and reproduction in any medium, provided the original work is properly cited.

Background. A high mortality has been reported during the first ninety days of cancer therapy and is more pronounced in patients with febrile neutropenia. The Bugando Medical Center oncology department offers cancer diagnosis and treatment services to the population of the Lake Zone of Tanzania with limited data on the outcome within 90 days of therapy. Here, we report the 90 -day mortality and factors associated with it among cancer patients attending the oncology department of the tertiary hospital in Tanzania. Methodology. Enrolled participants underwent baseline physical examinations, and their functional status was assessed using Karnofsky score. On each clinic visit, full blood picture was taken and patients were investigated for infections. Data were entered in the Microsoft Excel, cleaned and coded and then transferred to STATA version 13 for analysis. Results. A total of 102 participants were included in the final analysis. Their median age was 50 years (38-60). The majority of the study participants were females $76(75 \%)$, and $82(80.4 \%)$ had primary school education. The majority of the patients had solid cancer $96(94.1 \%)$. A total of $12(11.8 \%)$ patients died within 90 days of starting therapy. Low hemoglobin level at the start of cancer therapy, Karnofsky score below $80 \%$, and using 5-fluorouracil-containing therapy were statistically significantly found to be associated with mortality within 90 days of therapy among cancer patients. Conclusion. One tenth of cancer patients at Bugando Medical Center do not survive within 90 days of therapy; the mortality is significantly high among anemic patients, with poor performance status, on 5-fluorouracil regimen, and diagnosed with head and neck cancer, necessitating close follow-up of these patients.

\section{Background}

Cancer is the second leading cause of death worldwide [1], with more than $60 \%$ of cases being reported from Africa, Asia, and Central and South America. These regions account for about $70 \%$ of cancer deaths worldwide [2]. It was estimated that between 2000 and 2020 there would be $75 \%$ increase of cancer incidence in resource-limited countries [3]. Mortality rate due to cancer is more in resource-limited countries than in developed countries [4]. It is estimated that by 2020 , about $70 \%$ of deaths associated with cancer diseases would be from resource-limited countries and cancer survival rate would be $50 \%$ less than that of the developed countries [5].

In Tanzania, by the year 2008, there were 21,180 new cases of cancer per 42.5 million people [6]. In 2014, deaths due to cancers were reported to be 9,100 and 10,800 for men and women, respectively [7]. Cervical and breast cancers were leading causes of death among women while esophagus and head and neck cancers were leading causes of death 
among men [7]. Mortality among cancer patients is reported to be significantly high within the first 90 days of therapy [8, 9], and the incidence is reported to be 10.3 times higher in patients with one comorbidity (renal disease, heart disease, chronic bronchitis, or mucositis) than those without comorbidity and can increase to 24.1 times higher with the increase in number of comorbidities [8]. Other factors associated with mortality among cancer patients on therapy include the type of cancer, anticancer regimen, age, performance status, anemia, and neutropenia $[8,10,11]$. Furthermore, outpatients have been reported to have higher mortality rate than the inpatients which is associated with the lack of supportive care among outpatients $[8,10]$.

The number of cancer patients receiving medication at the oncology department of Bugando Medical Center (BMC) has increased due to the availability of chemotherapy, diagnostic techniques, and improved infrastructure for cancer management. However, data on mortality and its associated factors within 90 days of starting chemotherapy is still limited. Here, we are reporting mortality and factors associated with it within 90 days of therapy among cancer patients attending the oncology department of BMC. The spectrum of bacterial pathogens causing infections among these patients has also been determined.

\section{Methods}

This was a hospital-based cross-sectional study with a followup component which was conducted from August 2017 to May 2018 at the BMC oncology department. The unit attends about 100-150 cancer patients per month and receives up to 25 new patients per month (http://www .bugandomedicalcentre.go.tz/index.php? bmc=24). The study enrolled all patients with age above 12 years, histologically confirmed to have cancer and scheduled to start chemotherapy.

The minimum number of patients to be enrolled in the study was obtained using Kish and Lisle formula of 1965 [12]. The prevalence of mortality among cancer patients of $8 \%$ was substituted, and the minimum number of patients obtained was 82 [9].

2.1. Data Collection. A total of 114 patients were eligible to start cancer therapy at the BMC oncology department during the study period; however, we enrolled 111 patients because 3 patients declined participation.

At enrollment, physical and clinical examination was done; blood pressure was measured using a digital blood pressure monitor CHU 304 (Citizen Systems Japan Co. Ltd.) that has been clinically validated by the European Society of Hypertension protocol of 2010. Body temperature was measured by a digital thermometer MDD 93/42/EEC, “0197" (Holding Corp. GmbH (Hamburg)), and body weight and height were measured using DETECTO machine (Webb City, MO, USA). The data on type, stage of cancer, treatment regimen, and duration of therapy were recorded from the patients' file. At each visit, patients were evaluated for signs and symptoms of infections, especially fever.
At baseline, the following investigations were done: full blood picture (FBP), and urine and blood culture as per the BMC management protocol. Another FBP was taken during follow-up visits, and for patients who developed signs and symptoms of infections, blood and urine were collected for culture following the standard operating procedures of the accredited microbiology laboratory of BMC. Blood cell counts were analyzed by the use of Cell-Dyn 1800 automated hematology analyzer (Abbott Diagnostics, Illinois, USA). Drug susceptibility testing was done using Kirby Bauer disk diffusion technique and interpreted using the Clinical and Laboratory Standards Institute (CLSI) guidelines [13].

2.2. Data Management and Analysis. Laboratory data were recorded in a laboratory workbook and then entered onto the Excel spreadsheet for cleaning and coding before being transferred to STATA version 13 for analysis. Continuous data like blood pressure, body weight, Karnofsky score, temperature, white blood cell count, and neutrophil count were presented as medians and interquartile ranges and compared by the Wilcoxon rank-sum test. Categorical variables such as sex, marital status, occupation, and level of education were presented as frequency (percent) and compared by chisquare or Fisher's exact test. A $p$ value of $<0.05$ was considered statistically significant.

\section{Ethics Approval and Consent to Participate}

The protocol for this study was ethically approved by the joint CUHAS/BMC ethics and scientific review committee with certificate number CREC/228/2017. Permission to carry out the study was also sought from the management of the BMC and the oncology department. Written informed consent was obtained from the participants after being informed about the study objectives and procedures. For all participants aged less than 18 years, consents were sought from parents/guardians and assent was requested from the participant.

\section{Results}

4.1. Social Demographic and Baseline Clinical Data. Out of 111 enrolled patients, 102 were included in the final analysis. A total of 5 patients could not start cancer therapy due to financial reasons; two were lost to follow-up, one died before therapy was initiated and one shifted to a referral cancer hospital (Ocean Road Cancer Institute, Dar es Salaam, Tanzania).

The median age (IQR) of the study population was 50 (30-60) years. The majority were females $(76,74.5 \%)$, and (82 $80.3 \%$ ) had primary school education. The majority $(70,68.6 \%)$ were married. The majority of patients had solid cancers $(94.1 \%)$, and about one-third of patients reported at the oncology department while at cancer stage IV. All patients in stage IV of cancer received palliative care and low-dose chemotherapy calculated based on their performance status. Regarding various clinical parameters, the median body temperature (IQR) was $36.2^{\circ} \mathrm{C}$ (35.6-36.6), with median neutrophil (IQR) count of $3.0 \times 10^{9} / \mathrm{L}(2.1-4.1)$ and 
TABLE 1: Description of type of cancer seen during the study period.

\begin{tabular}{|c|c|c|}
\hline Variable & Frequency $(N)$ & Percent (\%) \\
\hline Gynecological cancers & 47 & 46 \\
\hline Cancer of the cervix & 40 & 39 \\
\hline Uterine cancer & 5 & 4.9 \\
\hline Ovarian cancer & 1 & 1 \\
\hline Choriocarcinoma & 1 & 1 \\
\hline Head and neck cancers & 12 & 12 \\
\hline Squamous cell carcinoma of the head and neck & 5 & 4.9 \\
\hline Adenocarcinoma of the head and neck & 3 & 2.9 \\
\hline Retinoblastoma & 2 & 2 \\
\hline Nasopharyngeal cancer & 1 & 1 \\
\hline Sinonasal cancer & 1 & 1 \\
\hline Connective tissue cancers (sarcoma) & 12 & 12 \\
\hline Kaposi’s sarcoma & 6 & 6 \\
\hline Malignant melanoma & 2 & 2 \\
\hline Malignant phyllodes & 1 & 1 \\
\hline Fibrosarcoma & 1 & 1 \\
\hline Osteosarcoma & 1 & 1 \\
\hline Chondrosarcoma & 1 & 1 \\
\hline Breast cancer & 11 & 11 \\
\hline Gastrointestinal cancers & 10 & 10 \\
\hline Hepatocellular cancer & 4 & 4 \\
\hline Pancreatic cancer & 2 & 2 \\
\hline Colon cancer & 2 & 2 \\
\hline Esophageal cancer & 1 & 1 \\
\hline Biliary rhabdomyosarcoma & 1 & 1 \\
\hline Hematolymphoid malignancies & 6 & 6 \\
\hline Hodgkin lymphoma & 2 & 2 \\
\hline Chronic myeloid leukemia & 1 & 1 \\
\hline Acute myeloid leukemia & 1 & 1 \\
\hline Burkitt's lymphoma & 1 & 1 \\
\hline Non-Hodgkin lymphoma & 1 & 1 \\
\hline Genital and reproductive cancer & 2 & 2 \\
\hline Perianal cancer & 1 & 1 \\
\hline Prostate cancer & 1 & 1 \\
\hline Lung cancer & 1 & 1 \\
\hline
\end{tabular}

median $\mathrm{Hb} 11.6 \mathrm{~g} / \mathrm{dL}$ (10.2-12.6). Out of 102 patients, 19 (18.6\%) were HIV positive.

4.2. Types of Cancer Diagnosed. The commonest type of cancer diagnosed was gynecological cancer $(47,46.1 \%)$ which was led by cervical cancer $(40,39 \%)$, followed by head and neck cancer $(12,11.8 \%)$ led by squamous cell carcinoma of the head and neck $(6,4.9 \%)$. Breast cancer was diagnosed in $11(10.8 \%)$ (Table 1$)$.

4.3. Mortality within 90 Days of Starting Chemotherapy. Mortality within 90 days of starting therapy among cancer patients at the oncology department was $12(11.8 \%)$. The age of those who died ranged between 33 and 76 years. All patients who died in the current study had solid cancer and anemia at baseline with hemoglobin range from 7.3 to $11.4 \mathrm{~g} / \mathrm{dL}$. The majority $(11,91 \%)$ reported at the oncology department at advanced stage, i.e., cancer stages 3 and 4 . A total of $9(75 \%)$ had a performance status below $80 \%$ by Karnofsky score (Table 2).

4.4. Culture Results and Mortality within 90 Days of Starting Chemotherapy. Among the 102 nonrepetitive blood cultures done at baseline, 7 (6.9\%) were culture positive. S. aureus $(4,3.9 \%)$ was the most frequently detected pathogen, followed by E. coli and Pseudomonas aeruginosa, 2 (1.9\%) and 1 (1\%), respectively. Regarding urine culture, 13/102 


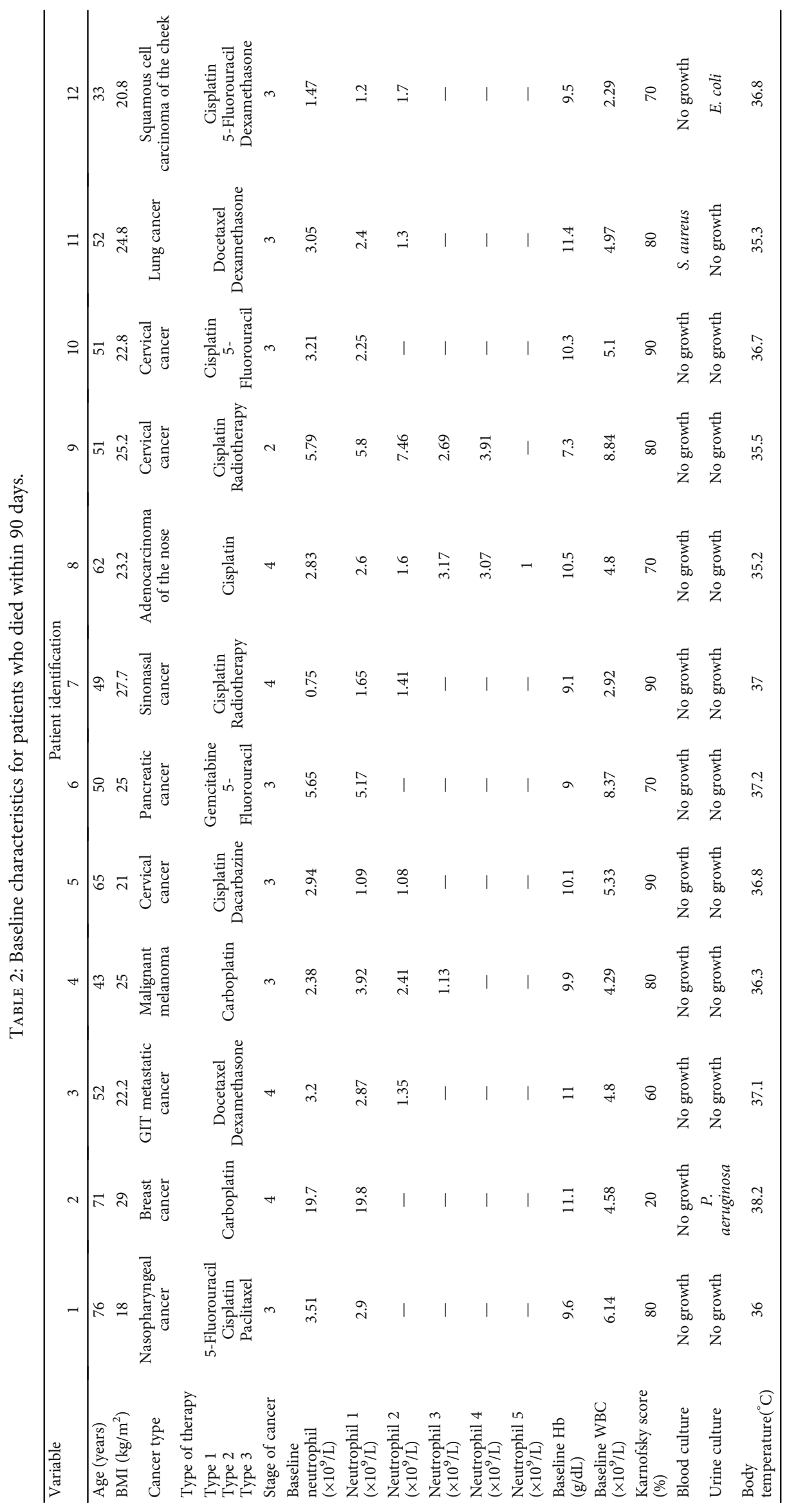


TABLE 3: Factors associated with mortality within 90 days of therapy.

\begin{tabular}{|c|c|c|c|}
\hline Variable & $\begin{array}{c}\text { Dead (12) } \\
\text { Number (\%) median [IQR] }\end{array}$ & $\begin{array}{c}\text { Alive (90) } \\
\text { Number (\%) median [IQR] }\end{array}$ & $p$ value \\
\hline \multicolumn{4}{|l|}{ HIV status } \\
\hline Unknown (21) & $3(14.3)$ & $18(85.7)$ & \\
\hline Negative (62) & $9(14.5)$ & $53(85.5)$ & \\
\hline Positive (19) & $0(0)$ & $19(100)$ & 0.211 \\
\hline Baseline body temperature $\left({ }^{\circ} \mathrm{C}\right)$ & $36.2[35.4-36.4]$ & $36.2[35.7-36.7]$ & 0.4023 \\
\hline BMI & 23.8 [21.7-25] & 22.9 [20.2-25.9] & 0.453 \\
\hline \multicolumn{4}{|l|}{ Cancer type } \\
\hline Others (90) & $8(8.9)$ & $82(91.1)$ & \\
\hline Head and neck (12) & $4(33.3)$ & $8(66.67)$ & 0.014 \\
\hline \multicolumn{4}{|l|}{ Therapy type } \\
\hline Cisplatin (50) & $7(14.0)$ & $43(86.0)$ & 0.492 \\
\hline 5-Fluorouracil (10) & $4(40.0)$ & $6(60.0)$ & 0.004 \\
\hline Paclitaxel (22) & $1(4.5)$ & $21(95.5)$ & 0.235 \\
\hline Carboplatin (9) & $2(22.2)$ & $7(77.8)$ & 0.308 \\
\hline Dacarbazine (4) & $1(25.0)$ & $3(75)$ & 0.402 \\
\hline Docetaxel (6) & $2(33.3)$ & $4(66.7)$ & 0.091 \\
\hline Karnofsky score (\%) & $80[70-85]$ & $90[80-90]$ & 0.001 \\
\hline Baseline $\mathrm{Hb}(\mathrm{g} / \mathrm{dL})$ & $10[9.3-10.8]$ & $11.8[10.5-12.7]$ & 0.001 \\
\hline Baseline white blood cell $\left(\times 10^{9} / \mathrm{L}\right)$ & $4.9[4.4-5.7]$ & $5.2[4-6.7]$ & 0.226 \\
\hline Baseline neutrophil $\left(\times 10^{9} / \mathrm{L}\right)$ & $3.1[2.6-4.6]$ & $3[2-4.1]$ & 0.653 \\
\hline \multicolumn{4}{|l|}{ Body temperature visit $\left({ }^{\circ} \mathrm{C}\right)$} \\
\hline Visit 1 & $36.6[36.1-37]$ & $36.4[35.9-36.8]$ & 0.852 \\
\hline Visit 2 & $36.7[36-36.9]$ & $36.2[35.8-36.8]$ & 0.677 \\
\hline Visit 3 & $36.1[36.1-36.3]$ & $36.3[35.8-36.8]$ & 0.565 \\
\hline Visit 4 & $36.3[35.5-37.1]$ & $36.2[35.7-36.6]$ & 0.758 \\
\hline Visit 5 & $35.2[35.2-35.2]$ & 36.4 [35.9-37] & 0.490 \\
\hline
\end{tabular}

(12.7\%) patients had significant bacteriuria at baseline. $S$. aureus $(7,6.9 \%)$ was the most frequently isolated bacteria species, followed by E. coli $(5,4.9 \%)$ and Pseudomonas aeruginosa $(1,1 \%)$. Of the 18 patients with bacterial infections, either UTI or blood stream infections, $3 / 18(16.6 \%)$ died compared to $9 / 84(10.8 \%)$ without bacterial infection, $p=$ 0.244 . Of the patients with bacterial infections who died, one had S. aureus blood stream infection and two had significant bacteriuria, one due to $P$. aeruginosa and another due to E. coli.

4.5. Factors Associated with Mortality. Significant proportion of patients with head and neck cancer died compared to patients with other types of cancer $(33.3 \%(4 / 12)$ vs. $8.9 \%$ $(8 / 90), p=0.014)$. The median hemoglobin level of patients who died was significantly lower than the median hemoglobin level of patients who survived $(10(9.3-10.8) \mathrm{g} / \mathrm{dl}$ vs. $11.8(10.5-12.7) \mathrm{g} / \mathrm{dl}, p=0.001)$ (Table 3).

\section{Discussion}

Mortality within 90 days of starting chemotherapy among cancer patients in the current study was found to be $12 \%$. The mortality reported was almost similar to $11 \%$ that was reported by Kuderer et al. from the United States among cancer patients on therapy in 2006 and slightly higher than $8 \%$ which has recently been reported from South Africa $[8$, 14]. Furthermore, the mortality was significantly high compared to $7.2 \%$ reported by the International Agency for Research on Cancer from Sub-Saharan Africa in the global cancer statistics of 2012 [15]. Additionally, similar mortality was reported in the study done in West Africa 8 years ago which reported the mortality of $10 \%$ [16]. Low functional status due to cancer itself and the toxicity brought by the therapy collectively compromise the health of the patients and might lead to high mortality observed after therapy initiation in the current study.

As previously reported in the world cancer report of 2012 [17], the current study found solid cancers to be the predominant cancer type at BMC. However, contrary to the world report which observed the commonest solid cancer to be lung cancer $[18,19]$, this study observed cervical cancer to be the commonest solid cancer. In Tanzania, cervical cancer has been the leading cancer in the past one decade $[20,21]$. The ongoing national campaign regarding screening for cervical cancer could explain the findings. Furthermore, it should be noted that maybe lung cancer is underreported due to the little attention that is given to it. 
Contrary to high mortality that has been reported in several studies $[8,22]$ among patients with hematological malignancies, in the current study, all patients with hematological malignancy survived within 90 days of therapy. Hematological malignancy is known to have higher mortality than solid cancer, the reason being that hematological malignancies are associated with both depletion and impaired function of neutrophils due to cancer itself and the toxicity of therapy [22, 23]. The mortality data for patients with hematological malignancy in the current study should be carefully interpreted due to the limited number of patients.

In the current study, severe neutropenia was not observed. Neutropenia has been associated with increased mortality in many studies $[22,23]$. In the current study, the use of granulocyte colony-stimulating factors among patients with reduced number of neutrophil before starting therapy prevented severe neutropenia hence reduced mortality of these patients.

As in a previous study in the United States and Greece, significantly more mortality was observed among patients in 5-fluorouracil treatment [24]. The toxicity induced by 5fluorouracil such as stomatitis, mucositis, and diarrhea [24] could partly explain the observation. As observed in a previous study in France [25], patients with carcinoma of the head and neck had two times odds of dying than patients with other cancers.

This study observed increased mortality in head and neck cancer patients; this could be due to the fact that the disease is close to vital organs with possibility of impairment in swallowing and breathing [26]. Additionally, significantly high mortality among patients with head and neck cancers in this study could be contributed by late stage presentation at the oncology department, advanced age, and poor performance status. Late stage presentation has been documented as the main predictor of mortality [26]. Furthermore, advanced age has been noted as the poor prognostic factor among patients with head and neck cancer [27].

As it was documented in a meta-analysis where anemia was found to be the leading cause of mortality [28], in the current study, all patients who died had anemia at the baseline. This could be due to the fact that anemia leads to the hypoxic state which reduces the amount of drug delivered to the cell and hence reduces the therapy response $[29,30]$. The findings of this study regarding high mortality among anemic patients confirm what has been reported in previous studies [28, 31]. Furthermore, anemia is associated with fatigue which causes the inability to perform daily activities among cancer patients and hence reduces the quality of life of patients and increases mortality [29, 30].

\section{Conclusion}

The mortality of cancer patients within 90 days of therapy was slightly higher than that of patients in Sub-Saharan Africa and more pronounced in patients with anemia at baseline, use of 5-fluorouracil, poor performance status, and diagnosis of head and neck cancers necessitating close follow-up of these patients. Furthermore, a study focused on hemato- logical cancers from developing countries to provide data on mortality is recommended.

\section{Abbreviations \\ BMC: Bugando Medical Centre \\ CLSI: Clinical and Laboratory Standards Institute \\ FBP: Full blood picture \\ IQR: Interquartile range.}

\section{Data Availability}

The data is available upon request and the request should be made to the Director of Research and Innovation, Catholic University of Health and Allied Sciences.

\section{Ethical Approval}

All procedures performed in studies involving human participants were in accordance with the ethical standards of the institutional and/or national research committee and with the 1964 Helsinki Declaration and its later amendments or comparable ethical standards. The study was given ethical clearance by the joint CUHAS/BMC ethics and scientific review committee with certificate number CREC/228/2017. Permission to carry out the study was also sought from the management of the Bugando Medical Centre and the oncology department.

\section{Consent}

Written informed consent was obtained from participants after being informed about the study objectives and procedures. For all participants aged less than 18 years, consents were sought from parents/guardians and assent was requested from the participant.

\section{Disclosure}

The funding body had no role in the design of the study and data collection, analysis and interpretation of the data, and in writing the manuscript.

\section{Conflicts of Interest}

The authors declare that they have no conflicts of interest.

\section{Authors' Contributions}

MJC, MFM, SEM, and BL designed the work. MJC, MFM, and VS recruited the patients and performed laboratory investigations. MJC and BL managed the patients. MFM, RK, and SEM analyzed and interpreted the data. MJC and MFM wrote the first draft of the manuscript which was critically reviewed by RK, VS, BL, and SEM. All authors read and approved the final version of the manuscript. 


\section{Acknowledgments}

The authors would like to acknowledge the support provided by the Department of Oncology of the Bugando Medical Centre and the technical support of Mr. Vitus Silago from the Department of Microbiology and Immunology, Catholic University of Health and Allied Sciences, Mwanza, Tanzania. This work was supported through the DELTAS Africa Initiative grant \# DEL-15-011 of THRiVE-2 to MJC. The DELTAS Africa Initiative is an independent funding scheme of the African Academy of Sciences' Alliance for Accelerating Excellence in Science in Africa (AESA) and supported by the New Partnership for Africa's Development Planning and Coordinating Agency (NEPAD Agency) with funding from the Wellcome Trust grant \# 107742/Z/15/Z and the UK Government.

\section{References}

[1] R. L. Siegel, K. D. Miller, and A. Jemal, "Cancer statistics, 2017," CA: a Cancer Journal for Clinicians, vol. 67, no. 1, pp. 7-30, 2017.

[2] M. Kurien, S. Sheelan, L. Jeyaseelan, Bramhadathan, and $\mathrm{K}$. Thomas, "Fine needle aspiration in chronic tonsillitis: reliable and valid diagnostic test," The Journal of Laryngology \& Otology, vol. 117, no. 12, pp. 973-975, 2003.

[3] D. M. Parkin, F. I. Bray, and S. S. Devesa, "Cancer burden in the year 2000. The global picture," European Journal of Cancer, vol. 37, pp. 4-66, 2001.

[4] M. P. Coleman, M. Quaresma, F. Berrino et al., "Cancer survival in five continents: a worldwide population-based study (CONCORD)," The Lancet Oncology, vol. 9, no. 8, pp. 730756, 2008.

[5] S. F. Sener and N. Grey, "The global burden of cancer," Journal of Surgical Oncology, vol. 92, no. 1, pp. 1-3, 2005.

[6] A. Jemal, M. M. Center, C. DeSantis, and E. M. Ward, "Global patterns of cancer incidence and mortality rates and trends," Cancer Epidemiology and Prevention Biomarkers, vol. 19, no. 8, pp. 1893-1907, 2010.

[7] Global Burden of Disease Cancer Collaboration, "Global, regional, and national cancer incidence, mortality, years of life lost, years lived with disability, and disability-adjusted lifeyears for 32 cancer groups, 1990 to 2015," JAMA Oncology, vol. 3, no. 4, pp. 524-548, 2017.

[8] N. M. Kuderer, D. C. Dale, J. Crawford, L. E. Cosler, and G. H. Lyman, "Mortality, morbidity, and cost associated with febrile neutropenia in adult cancer patients," Cancer, vol. 106, no. 10, pp. 2258-2266, 2006.

[9] M. E. R. O'Brien, A. Borthwick, A. Rigg et al., "Mortality within 30 days of chemotherapy: a clinical governance benchmarking issue for oncology patients," British Journal of Cancer, vol. 95, no. 12, pp. 1632-1636, 2006.

[10] G. H. Lyman, C. H. Lyman, O. Agboola, and for the Anc Study Group, "Risk models for predicting chemotherapy-induced neutropenia," The Oncologist, vol. 10, no. 6, pp. 427-437, 2005.

[11] L. A. Boxer, "How to approach neutropenia," Hematology. American Society of Hematology. Education Program, vol. 2012, no. 1, pp. 174-182, 2012.

[12] L. Kish, "Sampling organizations and groups of unequal sizes," American Sociological Review, vol. 30, no. 4, pp. 564-572, 1965.
[13] M. F. Mushi, A. E. Mwalutende, J. M. Gilyoma et al., "Predictors of disease complications and treatment outcome among patients with chronic suppurative otitis media attending a tertiary hospital, Mwanza Tanzania," BMC Ear, Nose and Throat Disorders, vol. 16, no. 1, 2016.

[14] F. Made, K. Wilson, R. Jina et al., "Distribution of cancer mortality rates by province in South Africa," Cancer Epidemiology, vol. 51, pp. 56-61, 2017.

[15] D. M. Parkin, F. Bray, J. Ferlay, and A. Jemal, "Cancer in Africa 2012," Cancer Epidemiology and Prevention Biomarkers, vol. 23, no. 6, pp. 953-966, 2014.

[16] V. Vanderpuye, J. Yarney, and K. Beecham, "Management of febrile neutropenia in patients receiving chemotherapy for solid tumors: a retrospective study of twenty cases from the radiotherapy centre, Accra, Ghana," West African Journal of Medicine, vol. 29, no. 5, 2011.

[17] L. A. Torre, F. Bray, R. L. Siegel, J. Ferlay, J. Lortet-Tieulent, and A. Jemal, "Global cancer statistics, 2012," CA: a Cancer Journal for Clinicians, vol. 65, no. 2, pp. 87-108, 2015.

[18] G. A. Alexander, "Geographical aspects of cancer in Tanzania," Journal of the National Medical Association, vol. 75, no. 8, pp. 797-804, 1983.

[19] F. Bray, J. Ferlay, I. Soerjomataram, R. L. Siegel, L. A. Torre, and A. Jemal, "Global cancer statistics 2018: GLOBOCAN estimates of incidence and mortality worldwide for 36 cancers in 185 countries," CA: A Cancer Journal for Clinicians, vol. 68, no. 6, pp. 394-424, 2018.

[20] J. Ferlay, I. Soerjomataram, M. Ervik et al., "GLOBOCAN 2012: estimated cancer incidence, mortality and prevalence worldwide in 2012," International Journal of Cancer, vol. 136, pp. E359-E386, 2012.

[21] M. Urasa and E. Darj, "Knowledge of cervical cancer and screening practices of nurses at a regional hospital in Tanzania," African Health Sciences, vol. 11, no. 1, pp. 48-57, 2011.

[22] T. W. LeBlanc, A. P. Abernethy, and D. J. Casarett, "What is different about patients with hematologic malignancies? A retrospective cohort study of cancer patients referred to a hospice research network," Journal of Pain and Symptom Management, vol. 49, no. 3, pp. 505-512, 2015.

[23] G. A. Curt, W. Breitbart, D. Cella et al., "Impact of cancerrelated fatigue on the lives of patients: new findings from the Fatigue Coalition," The Oncologist, vol. 5, no. 5, pp. 353-360, 2000.

[24] S. D'Andre, D. J. Sargent, S. S. Cha et al., “5-Fluorouracil based chemotherapy for advanced colorectal cancer in elderly patients: a north central cancer treatment group study," Clinical Colorectal Cancer, vol. 4, no. 5, pp. 325-331, 2005.

[25] S. S. Baxi, L. C. Pinheiro, S. M. Patil, D. G. Pfister, K. C. Oeffinger, and E. B. Elkin, "Causes of death in long-term survivors of head and neck cancer," Cancer, vol. 120, no. 10, pp. $1507-$ 1513, 2014

[26] J. Sarini, C. Fournier, J. L. Lefebvre, G. Bonafos, J. T. van, and B. Coche-Dequéant, "Head and neck squamous cell carcinoma in elderly patients: a long-term retrospective review of 273 cases," Archives of Otolaryngology-Head \& Neck Surgery, vol. 127, no. 9, pp. 1089-1092, 2001.

[27] J. A. Väisänen, A.-M. H. Syrjälä, P. R. O. Pesonen, M. J. Pukkila, P. T. Koivunen, and O.-P. Alho, "Characteristics and medical-care-seeking of head and neck cancer patients: a population-based cross-sectional survey," Oral Oncology, vol. 50, no. 8, pp. 740-745, 2014. 
[28] K. Knight, S. Wade, and L. Balducci, "Prevalence and outcomes of anemia in cancer: a systematic review of the literature," The American Journal of Medicine, vol. 116, no. 7, pp. 11-26, 2004.

[29] J. J. Caro, M. Salas, A. Ward, and G. Goss, "Anemia as an independent prognostic factor for survival in patients with cancer: a systemic, quantitative review," Cancer, vol. 91, no. 12, pp. 2214-2221, 2001.

[30] P. Harper and T. Littlewood, "Anaemia of cancer: impact on patient fatigue and long-term outcome," Oncology, vol. 69, no. 2, pp. 2-7, 2005.

[31] J. J. Caro, M. Salas, A. Ward, and G. Goss, "Anemia as an independent prognostic factor for survival in patients with cancer," Cancer, vol. 91, no. 12, pp. 2214-2221, 2001. 